\title{
Doenças Reumáticas e Infertilidade Masculina
}

\section{Rheumatic Diseases and Male Infertility}

\author{
Eutília Andrade Medeiros Freire ${ }^{(1)}$, Jamile de Castro Alves Nepomuceno( ${ }^{(2)}$, Inês de Oliveira Maia ${ }^{(2)}$, Rozana \\ Mesquita Ciconelli ${ }^{(3)}$
}

\section{RESUMO}

As doenças reumáticas podem causar distúrbios sexuais e reprodutivos. As razões destes distúrbios são multifatoriais. Manifestações e sintomas das doenças podem reduzir a libido e interferir no sucesso da reprodução, como ocorre na artrite reumatóide (AR), lúpus eritematoso sistêmico (LES), espondilite anquilosante (EA) e esclerose sistêmica (ES). A atividade da doença pode levar a uma alteração do eixo hipotálamo-hipófise, acarretando períodos de disfunção gonadal. Os auto-anticorpos e os distúrbios de hormônios sexuais, que podem estar presentes em muitas doenças reumáticas, podem influenciar negativamente na fertilidade. Além disso, algumas drogas usadas no tratamento de doenças reumatológicas por vezes representam um risco para a reprodução masculina, devido aos efeitos adversos que podem causar, como defeitos cromossômicos e gonadotoxicidade, prejudicando a espermatogênese e a motilidade dos espermatozóides. Conseqüentemente, há falência gonadal transitória ou permanente. Este trabalho faz uma revisão de um assunto pouco abordado na literatura brasileira.

Palavras-chave: doenças reumáticas, drogas anti-reumáticas, fertilidade masculina.

\section{INTRODUÇÃO}

As doenças reumáticas podem causar distúrbios nas funções sexual e reprodutiva. A atividade de doença pode levar a uma alteração do eixo hipotálamo-hipófise, acarretando períodos de disfunção gonadal. Os auto-anticorpos, que estão presentes em muitas doenças reumáticas, podem influenciar negativamente a fertilidade. Os efeitos tóxicos de drogas imunossupressoras podem induzir falência gonadal transitória ou permanente ${ }^{(1)}$.

\section{ALTERAÇÕES DA FERTILIDADE LIGADAS À DOENÇA}

Os distúrbios da função sexual ocorrem devido à interação de fatores físicos, emocionais e sociais associados à

\begin{abstract}
Rheumatic diseases affect all aspects of life, including sexuality and reproduction. The reasons for disturbing sexual functioning and reproduction are multifactorial. Manifestations and symptoms of disease can reduce libido and interfere with successful reproduction, for example, in rheumatoid arthritis $(A R)$, systemic lupus erythematosus (SLE), ankylosing spondylitis (AS) and systemic sclerosis (SSc). Active disease disturbs the hypothalamic-pituitary-axis, giving rise to periods of gonadal dysfunction. Autoantibodies, which are present in most of the rheumatic diseases, and disturbances of sex hormone status can negatively influence the fertilization. Furthermore, some antirheumatic drugs carry a risk for male reproduction, because can present adverse effects, which includes chromosomal defects and gonadotoxicity, damaging the spermatogenesis and sperm motility. It results in transient or permanent gonadal failure. We are presenting a review of this aspect.
\end{abstract}

Keywords: rheumatic diseases, anti-rheumatic drugs, man fertility.

doença, que contribuem para reduzir a atividade e o desempenho sexuais. Dor crônica, fadiga, rigidez e deterioração da função articular, assim como alterações psicológicas como depressão e baixa auto-estima, comuns em doenças crônicas, podem reduzir a libido e, conseqüentemente, a freqüência das relações sexuais. O prazer da relação pode diminuir devido à dor articular aos movimentos ou dificuldade de encontrar uma posição que não cause desconforto ${ }^{(2)}$.

Por outro lado, doenças reumáticas em atividade podem alterar o controle do eixo hipotálamo-hipófise, inibindo a secreção do hormônio liberador de gonadotropina e aumentando a secreção de prolactina, resultando em hipogonadismo ${ }^{(1-6)}$. A hiperprolactinemia interfere na espermatogênese e altera a motilidade e a qualidade dos

Departamento de Medicina Interna, Centro de Ciências da Saúde, Universidade Federal da Paraíba (UFPB), João Pessoa, PB. Recebido em 08/04/05. Aprovado, após revisão, em 24/10/05

1. Reumatologista, Professora Assistente da Disciplina de Reumatologia do Departamento de Medicina Interna da UFPB, Membro do Projeto Pronuclear da Sociedade Brasileira de Reumatologia (SBR).

2. Aluna da graduação em Medicina da UFPB.

3. Médica assistente, doutora da Disciplina de Reumatologia da Universidade Federal de São Paulo (UNIFESP), Membro do Projeto Pronuclear da SBR. Endereço para correspondência: Eutília Andrade Medeiros Freire, Rua José Gomes de Sá Filho, 135, apto. 403, Bessa, João Pessoa, CEP 58037-580, PB, Brasil, tel: (83) 3246-7826, e-mail: eutilia@terra.com.br 
espermatozóides $^{(6)}$. O resultado disso é a diminuição da libido, impotência, inabilidade para ejacular, inibindo a função sexual masculina. O hipogonadismo tem sido relatado em pacientes com artrite reumatóide (AR) e lúpus eritematoso sistêmico (LES) ${ }^{(3-5)}$. Entretanto, tal disfunção testicular não necessariamente diminui o desempenho sexual. Redução da libido, disfunção erétil e escassez ejaculatória têm sido relatadas em 19 a $35 \%$ dos homens com $\operatorname{LES}^{(7)}$. Silva et $a^{\left({ }^{8)}\right.}$ estudaram quatro adolescentes do sexo masculino com LES juvenil para avaliar a função gonadal. Foram realizadas avaliações clínica e laboratorial, com determinação de ultrasonografia testicular, FSH (hormônio folículo estimulante), LH (hormônio luteinizante), prolactina, testosterona e anticorpo antiesperma. Todos os pacientes forneceram três amostras de sêmen durante dois meses. Uma nova amostra foi coletada seis meses depois. A média de duração da doença foi de 6,6 anos. A idade média para a primeira ejaculação foi de 13,5 anos. Todos os pacientes tiveram doença grave com envolvimento renal. Todos relataram ereção e libido normais. A avaliação gonadal, através do exame da genitália e ultra-sonografia, foi normal. Anticorpos antiesperma foram negativos em todos os pacientes. Somente um paciente apresentou níveis séricos de FSH e LH elevados. As avaliações inicial e final do sêmen dos quatro pacientes foram anormais (azoospermia, oligoastenoteratospermia ou teratospermia). Apesar de esses pacientes apresentarem atividade sexual e genitália externa normais, a fertilidade deles pode estar diminuída, baseada nas anormalidades do esperma. Os autores concluem que análises seriadas do sêmen serão necessárias para classificar o grau e a duração das anormalidades do esperma em pacientes homens com LES.

Períodos de impotência sexual ocorrem em pacientes com AR e espondilite anquilosante $(\mathrm{EA})^{(3)}$. Em um número pequeno de casos, a EA crônica pode levar à síndrome da cauda eqüina com disfunção erétil, mas isto é muito $\operatorname{raro}^{(9)}$. Em homens com esclerose sistêmica (ES), a disfunção erétil pode ser decorrente de uma diminuição da pressão sangüínea no pênis causada por vasculite de pequenos vasos ${ }^{(10)}$. A varicocele pode ser de importância etiológica em até $33 \%$ dos casos de infertilidade masculina ${ }^{(11)}$. Ela é causada por fluxo retrógrado de sangue dentro da veia espermática interna que resulta em dilatação progressiva, freqüentemente palpável, do plexo venoso pampiniforme peritesticular. Acredita-se que resulte da incompetência da valva entre a veia espermática interna e a veia renal, sendo mais comum no lado esquerdo (85\%). A varicocele unilateral aumenta o fluxo sanguíneo e a temperatura em ambos os testículos, como resultado de extensas anastomoses dos sistemas ve- nosos. Acredita-se que o aumento da temperatura escrotal e testicular seja a causa da má qualidade do sêmen e da infertilidade. Os achados nas análises do sêmen geralmente são inespecíficos, com todos os parâmetros mostrando alguma anormalidade. O reparo cirúrgico da varicocele resulta em fertilidade em cerca de metade dos homens ${ }^{(11)}$. Em estudo comparativo entre pacientes com EA que não faziam uso de drogas anti-reumáticas modificadoras da doença (por exemplo, a sulfassalazina, que induz à infertilidade) e um grupo controle saudável, foi avaliada a incidência de varicocele ${ }^{(12)}$. Tanto os pacientes com EA como o grupo controle foram avaliados através de exame físico e Doppler colorido para varicocele. $\mathrm{O}$ diâmetro máximo das veias do plexo pampiniforme e a duração do refluxo foram avaliados. A amostra do sêmen também foi analisada em ambos os grupos. Os pacientes com EA tinham uma maior incidência de varicocele (52\%) quando comparados com o grupo controle (20\%). Os pacientes com EA tinham um aumento significativo do diâmetro das veias do plexo pampiniforme dos lados esquerdo e direito quando comparados com o do grupo controle. Portanto, a incidência da varicocele foi maior em pacientes com EA e a relevância clínica disso precisa ser avaliada. O trabalho citado não relata se os casos de EA eram avançados ou se havia comprometimento axial total. Pirildar et $a^{(13)}$ avaliaram a função sexual em 65 homens com EA, utilizando o Índice Internacional de Função Erétil (IIFE). Eles também observaram a freqüência e associação da disfunção erétil (DE) com a idade do paciente, duração da doença, rigidez matinal, atividade laboratorial, gravidade de doença, estado de depressão e medicação usada por esses pacientes. Comparado com o grupo controle saudável, os pacientes com EA apresentaram menores função erétil, número de relações sexuais e escores de satisfação de acordo com o IIFE. Não foram relatados achados clínico-laboratoriais para justificar a DE, exceto a duração da rigidez matinal. Os homens com o maior grau de rigidez matinal apresentaram escores de função erétil mais baixos que os homens com EA e com menor grau de rigidez matinal. Dos 65 pacientes com EA, 25 eram depressivos, enquanto não havia pacientes depressivos no grupo controle. Eles concluíram que a DE pode ocorrer no decurso da EA e que a patogênese da DE parece ser multifatorial, podendo estar associada à doença e ao tratamento preconizado.

Distúrbios de hormônios sexuais ocorrem em doenças reumáticas e podem reduzir a libido e interferir no sucesso da reprodução ${ }^{(14,15)}$. Estudos com andrógenos em homens com AR têm sido contraditórios. Alguns não encontraram nenhuma anormalidade nos níveis de andrógenos, outros 
relataram diminuição dos níveis de testosterona, deidroepiandrostenediona (DHEA) e sulfato de deidroepiandrostenediona (S-DHEA) ${ }^{(14,16,17)}$. Hipoandrogenicidade tem sido encontrada em homens com AR, especialmente na presença de atividade importante da doençą ${ }^{(16)}$. Concentrações séricas basais de testosterona total, globulina ligada ao hormônio sexual (SHBG) e LH foram medidos em 104 homens com AR e comparados com outros 99 homens saudáveis ${ }^{(17)}$. Os homens com AR tinham menores níveis de testosterona e, laboratorialmente, grande parte deles foi considerada portadora de hipogonadismo ${ }^{(16)}$.

Anormalidades auto-imunes têm sido associadas à infertilidade $^{(18)}$. No homem, anticorpos presentes no esperma têm sido associados à infertilidade, no entanto, somente os que estão direcionados aos espermatozóides podem inibir a reprodução. Um estudo não encontrou diferença na proporção de testes positivos para anticorpos antiesperma em pacientes com doenças reumáticas e o grupo controle ${ }^{(19)}$. A relação de anticorpos antiesperma associados à infertilidade permanece indefinida. Nos estudos de Marthur et a ${ }^{20)}$, títulos de anticorpos antiesperma determinados por hemaglutinação passiva e ensaios citotóxicos estiveram elevados em 62 homens e 46 mulheres de 103 casais com infertilidade primária (alterações testiculares) e em 15 homens e 12 mulheres de 25 casais com infertilidade secundária (deficiência de gonadotrofinas) ${ }^{(20,21)}$. Dos casais em que um ou ambos os parceiros tinham níveis elevados de anticorpos antiesperma, apenas quatro conseguiram a reprodução, porém todos tiveram aborto espontâneo, sendo que três tinham infertilidade secundária, e um dos homens apresentava oligospermia. Isto sugere um papel etiológico da auto-imunidade na infertilidade.

\section{ALTERAÇÕES DA FERTILIDADE LIGADAS AO TRATAMENTO}

Algumas drogas anti-reumáticas podem representar um risco para a reprodução masculina, devido aos efeitos adversos que podem causar, gonadotoxicidade e defeitos cromossômicos $^{(1)}$ (Tabela 1$)$.

\section{CICLOFOSFAMIDA}

A ciclofosfamida é um agente alquilante citotóxico. Pode alterar a função endócrina no homem, em particular, levando à falência gonadal com azoospermia, sendo encontrada em 50 a $90 \%$ dos homens tratados com ciclofosfamida ${ }^{(22,23)}$. O modo exato das ações citotóxica e imunossupressora da ciclofosfamida em nível celular não é completamente esclarecido. Os efeitos gonadotóxicos de drogas imunossupressoras
TABELA 1

DROGAS ANTI-REUMÁTICAS E REPRODUCÃO MASCULINA

\begin{tabular}{ll}
\hline Drogas & Alteração na fertilidade \\
\hline Ciclofosfamida & Sim, irreversível \\
\hline Clorambucil & Sim, irreversível \\
Sulfassalazina & Sim, reversível \\
\hline Metotrexato & Sim, reversível \\
\hline Colchicina & Sim, dados inconclusivos \\
\hline AINEs & Sim, reversível \\
\hline Inibidores de TNF & Não \\
Ciclosporina & Sim, reversível \\
\hline Azatioprina & Não \\
\hline
\end{tabular}

como a ciclofosfamida dependem do mecanismo de ação, dose e duração do tratamento ${ }^{(24)}$. A ciclofosfamida aumenta o risco de genotoxicidade por induzir aberrações cromossômicas ou mutações gênicas isoladas. Oligo ou azoospermia podem ser induzidas pela ciclofosfamida, podendo-se observar infertilidade irreversível após tratamento com esta droga. Os agentes alquilantes, além de causarem danos no epitélio germinativo testicular, também afetam a função das células de Leydig( ${ }^{(25)}$. Existem evidências de que a exposição às drogas resulta em dano ao genoma masculino e induz a mutações hereditárias na linhagem germinativa e más-formações ${ }^{(26)}$. A citotoxicidade desta droga é mediada pela alquilação do DNA na posição 7 da guanina e formação de crossing-over. $\mathrm{O}$ sistema reprodutor masculino é particularmente sensível a danos induzidos pela ciclofosfamida. O processo da espermatogênese é altamente comandado e regulado. As espermatogônias se dividem por mitose e formam os espermatócitos para iniciar o processo. Os espermatócitos originam, através de duas divisões meióticas, as espermátides. Durante a espermiogênese, as espermátides se diferenciam em espermatozóides. O tratamento com 1-2 $\mathrm{mg} / \mathrm{Kg}$ por mais de quatro anos aumenta a incidência da azoospermia e oligospermia em pacientes adultos do sexo masculino. Os autores do estudo não caracterizaram a via de administração. Estudos anteriores têm mostrado que o tratamento crônico com ciclofosfamida em ratos machos causa dano ao DNA dos espermatozóides e resulta em anormalidades na descendência. Os efeitos da ciclofosfamida na progênie são tempo-dose dependentes. $\mathrm{O}$ tratamento crônico com ciclofosfamida altera a histologia e a bioquímica do epitélio do epidídimo, mas a espermatogênese não é interrompida no rato. Entretanto, espermatozóides anormais são encontrados no lúmen, tanto do epidídimo como do testículo, após a exposição crônica à ciclofosfamida. 
Identificar os efeitos da ciclofosfamida na expressão gênica de células germinativas pode esclarecer os mecanismos envolvidos na infertilidade masculina mediada pela toxicidade desta droga ${ }^{(26)}$. Reparo do DNA, proteínas de choque térmico, e mecanismos de defesa antioxidantes representam um importante papel na resposta à ação de agentes alquilantes e no desenvolvimento e função de células germinativas masculinas. Em embriões de ratos durante a organogênese e em células epiteliais tímicas de ratos ou em células tumorais, a ciclofosfamida induz à apoptose ${ }^{(22)}$. Em estudos anteriores, foi demonstrado que a ciclofosfamida tem efeitos estágioespecíficos nas células germinativas masculinas: células germinativas que foram pioneiras na exposição à ciclofosfamida, como espermátides e espermatozóides, produziram efeitos adversos na progênie, que incluíram letalidade dominante e más-formações. Existem evidências de que a ciclofosfamida induz danos ao DNA de células germinativas e que algumas dessas células são removidas por apoptose, resultando na redução do número de espermatozóides maduros (oligospermia ou azoospermia).

Durante o tratamento com ciclofosfamida, contracepção segura é mandatória em homens e mulheres, e a gravidez deve ser postergada para até três meses após a suspensão do medicamento devido à possibilidade de mutagenicidade ${ }^{(1)}$. O risco de infertilidade permanente, após tratamento com ciclofosfamida, pode ser evitado com a criopreservação de espermatozóides antes de iniciar o tratamento.

Um estudo realizado na Universidade de Sassari, na Itália, teve como objetivo avaliar o possível efeito protetor da testosterona, a partir da inibição da atividade das células germinativas masculinas expostas à ciclofosfami$\mathrm{da}^{(23)}$. A testosterona induz azoospermia ou oligospermia grave por reduzir os níveis de gonadotrofina e testosterona intragonadal. Assim, a testosterona pode diminuir a sensibilidade das células germinativas aos efeitos tóxicos da ciclofosfamida. Os pacientes foram divididos em três grupos. Os cinco pacientes do grupo A (três com nefrite membranosa, um com nefrite mesangial e um com nefrite lúpica) receberam ciclofosfamida oral (dose $150 \mathrm{mg} /$ dia) por seis a oito meses. Os cinco pacientes do grupo B (dois com nefrite membranosa, dois com nefrite mesangial e um com glomerulonefrite focal) e os cinco do grupo C (dois com nefrite membranosa, um com nefrite mesangial, dois com nefrite lúpica) receberam ciclofosfamida intravenosa (IV) em bolus de $15 \mathrm{mg} / \mathrm{Kg}$ mensalmente por oito meses. Pacientes do grupo C também receberam testosterona, $100 \mathrm{mg}$ (IM) a cada 15 dias. Os pacientes começaram a receber testosterona 30 dias antes de iniciar o tratamento com ciclofosfamida e continuaram recebendo durante todo o tratamento. Para assegurar a possibilidade de futura paternidade, amostras de sêmen de cada paciente foram coletadas antes de iniciar o tratamento. Amostras de sêmen também foram coletadas durante o terceiro e sexto meses de tratamento e, no terceiro e sexto meses depois do final do tratamento. Todas as amostras de sêmen foram analisadas no mesmo laboratório e com a mesma técnica. Amostras séricas de LH e FSH foram coletadas nos mesmos intervalos das amostras do sêmen. Os pacientes dos grupos A e $\mathrm{B}(\mathrm{n}=10)$ que não receberam testosterona desenvolveram azoospermia durante o tratamento com ciclofosfamida. Em um dos dez pacientes, a contagem de espermatozóides retornou ao normal seis meses depois do fim da terapia. Os níveis de FSH estavam elevados nesses pacientes. Todos os cinco que receberam testosterona desenvolveram azoospermia ou oligospermia grave durante o tratamento, mas tiveram uma contagem de espermatozóides normal seis meses depois do fim do tratamento. Nesses pacientes, os níveis de FSH eram normais. Os achados desse estudo confirmam que o tratamento com ciclofosfamida pode afetar a função das células germinativas no homem. Todos os pacientes $(\mathrm{n}=10)$ que receberam ciclofosfamida oral diariamente e quatro dos cinco que receberam mensalmente bolus (IV) de ciclofosfamida tiveram azoospermia por até seis meses depois do fim da terapia imunossupressora. Níveis séricos de FSH estiveram elevados em todos os pacientes que tiveram azoospermia, um achado que reflete uma alteração grave do epitélio germinativo. Este achado indica que o uso da testosterona, que inibe a atividade das células germinativas, pode proteger contra os efeitos da ciclofosfamida. A função das células de Leydig não foi afetada em nenhum dos pacientes, demonstrado pelos níveis normais de LH. Estudos prévios demonstraram que as células de Leydig são mais resistentes que as células germinativas à ação dos agentes alquilantes. Este estudo concluiu que a administração de testosterona antes e durante o tratamento com ciclofosfamida pode preservar a fertilidade masculina. Em estudo realizado pela $M c$ Gill University, foi observada a ocorrência de apoptose célula-específica em células germinativas masculinas, depois da retirada de testosterona ${ }^{(22)}$.

\section{CLORAMBUCIL}

O clorambucil é outro agente alquilante que pode levar à infertilidade irreversível. Em um estudo realizado por Tabbara $^{(27)}$, dez pacientes com doença de Behçet foram tratados somente com clorambucil. Sete destes pacientes desenvolveram oligospermia e três deles, azoospermia. Em 
um dos pacientes, que tinha 30 anos de idade, foi realizada uma biópsia testicular dois anos depois do fim do tratamento. O histopatológico revelou evidência de atrofia testicular. Por isso, durante o tratamento com clorambucil, é importante uma contracepção segura para homens e mulheres, e deve-se evitar gravidez até três meses após a suspensão do medicamento, devido à possibilidade de mutagenicidade.

\section{SULFASSALAZINA}

A sulfassalazina, uma droga muito utilizada no tratamento das artropatias inflamatórias soronegativas, tem sido associada à infertilidade masculina ${ }^{(28)}$. Esse efeito é atribuído mais à sulfapiridina que ao ácido-5-amino-salicílico, o componente ativo e porção antiinflamatória desta droga. A sulfassalazina causa oligospermia, além de reduzir a motilidade dos espermatozóides e aumentar a proporção de formas anormais dos mesmos. A Universidade de Panjab realizou um estudo com o objetivo de determinar os efeitos da sulfassalazina na fertilidade de ratos machos ${ }^{(29)}$. Foi administrado sulfassalazina $(120,250$ e $500 \mathrm{mg} / \mathrm{kg}$ ) via oral por 60 dias aos ratos sexualmente maduros. Tanto a motilidade dos espermatozóides como a fertilidade dos animais foram significativamente alteradas na administração da dose mais alta $(500 \mathrm{mg} / \mathrm{Kg})$. Anormalidades morfológicas incluíram alteração do perfil normal dos espermatozóides e presença de lesões na cabeça dos espermatozóides. Os resultados desse estudo sugerem que a sulfassalazina pode induzir à infertilidade, alterando a motilidade, forma e o número de espermatozóides. O efeito tóxico direto da sulfassalazina na maturação das células germinativas masculinas é aparente, mas está associado à influência de outros fatores, como à doença de base e a diversas formas de terapia, tal como, os esteróides, que também influenciam a espermatogênese ${ }^{(30)}$. O mecanismo de ação da sulfassalazina é ainda um assunto controverso. Os possíveis fatores que influenciam são: uma ação antifolato, que influencia na rapidez da divisão das células espermatogênicas, um efeito antiprostaglandina na motilidade e efeitos tóxicos da sulfapiridina. Um estudo realizado pela Chiba University teve como objetivo a investigação dos mecanismos que causam infertilidade em ratos tratados com sulfassalazina ${ }^{(31)}$. Esses animais receberam sulfassalazina na dose de $600 \mathrm{mg} / \mathrm{Kg}$ por 28 dias, avaliando-se a motilidade dos espermatozóides e a reação acrossômica. A capacitação e a reação acrossômica são dois processos de fundamental importância para a fertilização do ovócito com o espermatozóide ${ }^{(32)}$. Durante a capacitação, ocorrem alterações nas características da membrana, na atividade enzimática e nas propriedades da motilidade dos espermatozóides, que os tornam capazes de penetrar no ovócito, estimulando a reação acrossômica. A motilidade dos espermatozóides e a reação acrossômica foram significativamente reduzidas pela sulfassalazina ${ }^{(31)}$. Além disso, para investigar os mecanismos moleculares da infertilidade induzida pela sulfassalazina, foram interpretadas análises da expressão de mRNA nos testículos. Isso revelou que a molécula CD59, que fica localizada na membrana acrossômica e é importante para a função reprodutiva dos espermatozóides, foi afetada nos testículos, mas a espermatogênese não foi afetada. Esse estudo concluiu que os mecanismos moleculares fundamentais da infertilidade causada pela sulfassalazina não são ainda conhecidos.

\section{METOTREXATO}

O metotrexato é uma droga anti-reumática com atividade citotóxica que pode induzir à infertilidade reversível. Oligo ou azoospermia ocorre raramente durante a terapia com metotrexato, sendo reversível quando se retira a droga do tratamento ${ }^{(24)}$. Em um relato de caso, um jovem com psoríase grave apresentou oligospermia grave, que esteve presente durante a administração de metotrexato e pôde ser documentada pela análise seriada do fluido seminal ${ }^{(33)}$. A retirada desta droga resultou em celularidade normal de esperma. Medições dos níveis séricos de gonadotrofina e testosterona foram normais durante e depois do tratamento com metotrexato. Foi concluído, neste relato de caso, que a administração de metotrexato resultou em oligospermia grave e que estes efeitos inibitórios na espermatogênese foram reversíveis. Existem opiniões divergentes na literatura a respeito dos efeitos do metotrexato na fertilidade masculina ${ }^{(34)}$. Muitos relatos de caso e estudos não referem efeito adverso, outros referem esterilidade reversível após o uso desta droga. Uma limitação para muitos desses estudos é a administração concomitante de outros agentes quimioterápicos. Em estudo realizado pelo St. Louis University Medical Center, a toxicidade testicular foi avaliada em ratos através da infusão intravenosa de bolus de metotrexato ${ }^{(35)}$. A toxicidade testicular foi avaliada qualitativamente pela histologia, e quantitativamente pelo peso testicular, contagem de espermatozóides, escore modificado de Johnsen, índice de repopulação e índice epididimal. Oligospermia ocorreu com dosagens baixas e intermediárias de metotrexato, mas atrofia testicular não foi observada. A técnica da criopreservação de espermatozóide antes de iniciar o tratamento também é aconselhável em pacientes em uso de metotrexato, não devido à toxicidade gonadal, mas sim pela mutagenicidade ${ }^{(1)}$. 


\section{COLCHICINA}

Colchicina é usada no tratamento de várias doenças, incluindo a artrite gotosa, febre familiar do mediterrâneo (FFM) e doença de Behçet ${ }^{(36)}$. Como modulador dos microtúbulos no nível do citoesqueleto, esta droga detém a divisão celular na metáfase e inibe a motilidade celular dos microtúbulos. A motilidade dos espermatozóides depende da função microtubular e é concebível que a colchicina possa afetar a função dos espermatozóides. Muitas controvérsias existem em relação aos efeitos adversos da colchicina na produção e função dos espermatozóides, embora haja a impressão de que a ocorrência de patologia dos espermatozóides em pacientes com FFM, usando doses recomendadas de colchicina, é muito pequena. Análise dos espermatozóides mostrou um espectro de alterações, de oligo e azoospermia à normospermia com distúrbios na motilidade dos espermatozóides. Estas alterações inconsistentes dos espermatozóides podem ser explicadas porque, em pacientes com FFM, a progressão da doença pode induzir disfunção testicular, conseqüentemente, afetando a espermatogênese. Dessa maneira, parece que a colchicina por si só pode não ter um significativo efeito adverso direto na produção e função dos espermatozóides.

FFM é manifestada por episódios recorrentes de febre, serosite e artrite. Os sintomas geralmente começam antes dos 20 anos de idade. A amiloidose é uma das principais complicações desta doença. Profilaxia contínua com colchicina é efetiva tanto na supressão dos ataques da doença na maioria dos pacientes, como também na prevenção do desenvolvimento de amiloidose ${ }^{(37,38)}$. Em um estudo recente, análises de sêmen de seis pacientes com FFM, em tratamento crônico com colchicina, foram normais, assim como o perfil hormonal $^{(39)}$. Outro relato de 19 homens com FFM, que receberam colchicina, revelaram distúrbios da fertilidade em 4 deles durante o tratamento ${ }^{(37)}$. Esses relatos conflitantes podem sugerir que a expressão deste efeito adverso da colchicina em pacientes com FFM pode depender de outros fatores predisponentes, como a própria doença de base.

A associação entre patologia dos espermatozóides e amiloidose foi relatada em 11 homens jovens com AR complicada com amiloidose. Azoospermia, oligoteratoastenospermia e criptozoospermia foram encontrados em sete deles ${ }^{(25)}$. Depósitos amilóides foram detectados nos vasos testiculares de três desses pacientes. Recentemente, foram encontrados dois pacientes com FFM e azoospermia e que apresentavam amiloidose renal e uremia. Biópsia testicular desses dois casos mostrou depósitos amilóides nos vasos. Esta observação pode sugerir que o risco de azoospermia em pacientes com FFM associada à amiloidose e/ou à uremia é maior comparando-se com os casos que só apresentam FFM.

A eficácia da terapêutica da colchicina na gota é provavelmente devida à supressão da formação e liberação do fator quimiotático de neutrófilo ${ }^{(40)}$. Usualmente, infertilidade não diz respeito à maioria da população gotosa mais idosa. Entretanto, relatos recentes implicaram que o uso de colchicina pode levar a anormalidades dos espermatozóides $^{(41)}$. Azoospermia tem sido documentada durante o tratamento crônico de gota com colchicina. A dose administrada foi de $0,5 \mathrm{mg}$ duas vezes por dia durante três anos. Três meses depois do fim do tratamento, a contagem de espermatozóides voltou aos níveis normais.

Doença de Behçet é uma patologia multissistêmica, caracterizada por alterações ocular, mucocutânea, articular, gastrointestinal, neurológica e vascular ${ }^{(42)}$. A etiologia dessa doença é desconhecida. Os homens são três vezes mais afetados que as mulheres. O envolvimento urogenital é freqüente e inclui: epididimite, uretrite, cistites recorrentes e lesões aftosas genitais. A colchicina é usada para prevenir episódios de artrite e manifestações oculares. Em 131 pacientes com doença de Behçet que receberam $1 \mathrm{mg}$ de colchicina diariamente por 1 ano, oligozoospermia, entre outros efeitos colaterais, foi documentada em 11 deles ${ }^{(43)}$. Em um estudo adicional, a fertilidade de 31 pacientes com doença de Behçet que foram tratados com ciclofosfamida e/ou colchicina foi investigada pela análise do sêmen e pelos valores séricos de $\mathrm{FSH}^{(42)}$. $\mathrm{O}$ número de pacientes que receberam exclusivamente colchicina foi somente seis. A contagem de espermatozóides antes de iniciar o tratamento foi normal, sugerindo que a doença de base não impede a produção de espermatozóides. Os autores concluíram nesse estudo que a ciclofosfamida impede a espermatogênese, enquanto a colchicina, não. Recentemente, o acometimento urológico e os efeitos da colchicina na fertilidade em pacientes com doença de Behçet foram avaliados em 62 homens ${ }^{(44)}$. Envolvimento urogenital foi identificado em 2/3 do grupo estudado, epididimite em $8 \%$ e fluxo sanguíneo arterial peniano anormal em $27 \%$ do grupo. Em contraste com o relato anterior, patologia dos espermatozóides foi detectada em um número significativo de pacientes com doença de Behçet que receberam colchicina: $37 \%$ dos homens tinham oligonecrospermia e 3\% tinham oligospermia. A dose diária de colchicina não foi mencionada, mas a deterioração da qualidade do esperma acelerou com o uso prolongado da colchicina. Epididimite e anormalidade no fluxo sanguíneo arterial foram também prevalentes com o avanço da doença. Os autores concluíram 
que estes achados também podem influenciar na patologia do esperma. A possível explicação pode estar associada ao efeito da vasculite e da epididimite na espermatogênese. Estas patologias de base podem predispor os pacientes a um maior risco de oligo ou azoospermia associado à administração de colchicina.

\section{ANTIINFLAMATÓRIOS NÃO-ESTEROIDAIS}

As drogas antiinflamatórias não-esteroidais (AINEs) agem, principalmente, na inibição da ciclooxigenase, que é a enzima catalisadora da síntese de prostaglandinas $(\mathrm{PG})^{(45)}$. No homem, a maior concentração de prostaglandinas é encontrada no fluido seminal ${ }^{(46)}$. Elas são hormônios eicosanóides que estão associados a efeitos na motilidade dos espermatozóides ${ }^{(47)}$. Kennedy $\mathrm{JH}^{(47)}$ cita em seu trabalho que Schlegel et al mostraram que a $\mathrm{PGF}_{2 \alpha}$ foi correlacionada negativamente com a motilidade dos espermatozóides humanos. Por outro lado, as PG da série $\mathrm{E}$ promovem um influxo de íon cálcio, que é capaz de induzir à reação acrossômica. Em geral, as PG da série E estimulam a motilidade dos espermatozóides, enquanto as $\mathrm{PGF}_{2 \alpha}$ inibem-na. Os efeitos da inibição da biossíntese de PG foram estudados em dois grupos de pacientes com oligospermia que apresentavam alto ou baixo-normal níveis de PG seminal ${ }^{(48)}$. Os principais parâmetros do líquido seminal (volume, concentração, motilidade e morfologia dos espermatozóides) foram avaliados ao fim do tratamento diário de $100 \mathrm{mg}$ de indometacina, via oral, por 30 dias. Uma queda dos níveis de PG foi observada em ambos os grupos de pacientes, porém somente no grupo com altas concentrações de prostanóide, derivado da inibição de prostaglandina, foi correlacionado com uma significativa melhora da contagem e motilidade de espermatozóides. Os efeitos de doses variadas de indometacina foram examinados em 100 pacientes com oligospermia ${ }^{(49)}$. Esse tratamento aumentou a contagem e motilidade de espermatozóides e a capacidade de fertilidade. $\mathrm{O}$ exame de radioimunoensaio mostrou um aumento sérico de FSH e LH, mas um decréscimo de testosterona. Os resultados mostraram que a influência da indometacina foi melhor na dose de $75 \mathrm{mg}$ por dia. $\mathrm{O}$ mecanismo dessas alterações não está esclarecido. Um outro estudo avaliou o efeito inibitório do diclofenaco sódico na síntese de prostaglandinas sobre a espermatogênese de pacientes com oligospermia ${ }^{(50)}$. $\mathrm{O}$ diclofenaco sódico foi administrado na dose de $100 \mathrm{mg}$, via oral, diariamente, por 30 dias. O grupo, que apresentou altos níveis de prostaglandinas seminal mostrou um significativo aumento do número e motilidade dos espermatozóides, ao contrário do grupo que apresentou níveis normais de prostaglandina seminal.

\section{INIBIDORES DO TNF}

Os anticorpos antifator de necrose tumoral são potentes agentes para o tratamento da doença de Crohn, AR e EA. O fator de necrose tumoral (TNF) tem efeitos pró-inflamatórios diversos na mucosa intestinal e na membrana sinovial, e é uma citocina essencial na cascata inflamatória ${ }^{(51)}$. O tratamento da AR com antagonistas TNF é altamente efetivo ${ }^{(52)}$. Essas drogas induzem a apoptose célula-específica de monócitos e macrófagos, sugerindo ser um caminho potencial para a redução da inflamação sinovial. Evidências apresentadas no estudo realizado por Bullimore ${ }^{(53)}$ sugerem que a produção do TNF- $\alpha$ está envolvida no estabelecimento e manutenção da endometriose e também é responsável pela infertilidade através do efeito sobre a motilidade dos espermatozóides e sobre a função e desenvolvimento do ovócito. Endometriose é um distúrbio ginecológico que causa dor, infertilidade e alterações emocionais. O TNF- $\alpha$ está presente em níveis elevados no líquido peritoneal de mulheres inférteis com endometriose. $\mathrm{O}$ efeito do TNF- $\alpha$ na motilidade dos espermatozóides humanos in vitro foi avaliado utilizando-se o fluido peritoneal de mulheres inférteis com endometriose, contendo $0,100,400$ ou $800 \mathrm{U}$ de $\mathrm{TNF} / \mathrm{ml}$ assim como concentrações similares de TNF- $\alpha$ humano recombinante. Não houve redução da motilidade na presença de TNF- $\alpha$ em concentrações de $100 \mathrm{U} / \mathrm{ml}$. Entretanto, 500 e $1000 \mathrm{U}$ de TNF- $\alpha$ recombinante causaram uma significativa redução na motilidade dos espermatozóides depois de 4 e 21 horas de incubação quando comparados com o grupo controle. Similarmente, o fluido peritoneal contendo $100 \mathrm{U}$ de TNF- $\alpha / \mathrm{ml}$ não reduziu significativamente a motilidade dos espermatozóides depois de 4 ou 21 horas de incubação, mas o líquido peritoneal contendo $400 \mathrm{U}$ de TNF- $\alpha / \mathrm{ml}$ reduziu progressivamente a motilidade dos espermatozóides depois de 4 e 21 horas e totalmente depois de 21 horas de incubação. A capacidade do TNF- $\alpha$ em causar uma significativa redução da motilidade dos espermatozóides in vitro sugere que este pode ser o mecanismo da infertilidade observada em mulheres com endometriose $^{(54)}$.

\section{CICLOSPORINA-A}

A ciclosporina-A é um imunossupressor que, além de hepato e nefrotoxicidades, pode causar toxicidade testicular. Massuda et al ${ }^{(55)}$ cita em seu trabalho que Seethalakshmi et al foram os primeiros a relatar que a administração de ciclosporina-A causava alterações degenerativas dos túbulos seminíferos e um decréscimo na 
contagem e motilidade dos espermatozóides, resultando em infertilidade masculina ${ }^{(55)}$. Masuda et a ${ }^{(55)}$ observaram que a administração de ciclosporina-A reduziu a atividade fagocítica da célula de Sertoli e levou às más-formações das espermátides ou à fragilidade dos espermatozóides. Entretanto, as alterações morfológicas ocorridas nas células espermatogênicas dentro dos túbulos seminíferos não são totalmente esclarecidas.

\section{AZATIOPRINA}

O tratamento com azatioprina parece ser seguro para homens que desejam a paternidade ${ }^{(56)}$. Em relatos de 57 gravidezes, com o pai exposto à azatioprina durante a concepção, 56 bebês nasceram normais e apenas 1 tinha um defeito do tubo neural.

\section{CONCLUSÕES}

O impacto das doenças reumáticas na função sexual não é rotineiramente valorizado pelos médicos e outros profissionais de saúde e raramente presentes em questionários de qualidade de vida. É importante que esses profissionais informem aos pacientes a natureza das doenças reumáticas e a possibilidade de alguns medicamentos afetarem a vida sexual, sugerindo possíveis soluções que possam aliviar a

\section{REFERÊNCIAS}

1. Ostensen $\mathrm{M}$ : New insights into sexual functioning and fertility in rheumatic diseases. Best Pract Res Clin Rheumatol 18: 219-32, 2004.

2. Panush RS, Mihailescu GD, Gornisiewicz MT, Sutaria SH, Wallace DJ: Sex and arthritis. Bull Rheum Dis 49: 1-4, 2000.

3. Gordon D, Beastall GH, Thomson JA, et al: Androgenic status and sexual function in males with rheumatoid arthritis and ankylosing spondylitis. Q J Med 60: 671-679, 1986.

4. Vilarinho ST, Costallat LTL: Evaluation of the hypothalamicpituitary-gonadal axis in males with systemic lupus erythematosus. J Rheumatol 20: 725-30, 1998.

5. Mok CC, Lau CS: Profile of sex in male patients with systemic lupus erythematosus. Lupus 9: 252-7, 2000.

6. De Rosa M, Zarrilli S, Di Sarno A, et al: Hyperprolactinemia in men: clinical and biochemical features and response to treatment. Endocrine 20: 75-82, 2003.

7. Sant SM, O'Connell D: Cauda equina syndrome in ankylosing spondylitis: a case report and review of the literature. Clin Rheumatol 14: 224-6, 1995.

8. Silva CA, Hallack J, Pasqualotto FF, Barba MF, Saito MI, Kiss $\mathrm{MH}$ : Gonadal function in male adolescents and young males with juvenile onset systemic lupus erythematosus. J Rheumatol 29: 2000-5, 2002.

9. Folomeev M, Alekberova Z: Impotence in systemic lupus ansiedade. Além disso, é essencial que se discuta um planejamento familiar no caso de o paciente desejar a paternidade durante ou logo após o período de tratamento, a fim de evitar maiores intercorrências gestacionais.

Durante o tratamento com drogas anti-reumáticas, é importante também a contracepção masculina e/ou feminina, para se evitar a mutagenicidade. Com relação aos métodos contraceptivos masculinos, a vasectomia é o mais seguro e que pode ser reversível por microcirurgia. $\mathrm{O}$ tratamento hormonal, através da reposição com testosterona associada a progestogênio, ainda não é considerado um método totalmente confiável e seguro ${ }^{(57)}$. No entanto, este assunto tem sido abordado em pesquisas clínicas e farmacológicas, com o objetivo de garantir um meio seguro, reversível e efetivo de contracepção ${ }^{(58)}$.

Na prática clínica, o reumatologista deve, então:

Esclarecer e informar ao paciente sobre os riscos do tratamento;

- Orientá-lo sobre a possibilidade de infertilidade transitória ou permanente;

Orientá-lo sobre a importância da contracepção masculina e/ou feminina durante o tratamento;

- Se há o desejo de procriação, orientá-lo sobre a possibilidade de criopreservação dos espermatozóides antes de começar a terapia.

erythematosus, J Rheumatol 17: 117-8, 1990.

10. Nowlin NS, Brick JE, Weaver DJ, et al: Impotence in scleroderma. Ann Intern Med 104: 794-8, 1986.

11. Griffin JE, Wilson JD: Distúrbios dos testículos. Harrison Medicina Interna, 15.a ed, Rio de Janeiro, Editora Mc Graw-Hill Interamericana do Brasil, 2002.

12. Ozgocmen S, Kocakoc E, Kiris A, Ardicoglu A, Ardicoglu O: Incidence of varicoceles in patients with ankylosing spondylitis evaluated by physical examination and color duplex sonography. Urology 59: 919-22, 2002.

13. Pirildar T, Muezzinoglu T, Pirildar S: Sexual function in ankylosing spondylitis: a study of 65 men. J Urol 171: 1609-10, 2004.

14. Mais AT: Hormonal and pregnancy relationships to rheumatoid arthritis: convergent effects with immunological and microvascular systems. Semin Arthritis Rheum 25: 1-27, 1995.

15. Cutolo M, Balleari E, Giusti M, et al: Sex hormone status in women suffering from rheumatoid arthritis. Rheumatology 13 : 1019-23, 1986.

16. Gordon D, Beastall GH, Thomson JA, et al: Prolonged hypogonadism in male patients with rheumatoid arthritis during flares in disease activity. Br J Rheumatol 27: 440-44, 1988.

17. Tengstrand B, Carlstrom K, Hafstrom I: Bioavailable testosterone in men with rheumatoid arthritis high frequency of hypogonadism. Rheumatol 41: 285-89, 2002. 
18. Ghazeeri GS, Kutteh WH: Autoimmune factors in reproductive failure. Current Opinion in Obstet Gynecol 13: 287-91, 2001.

19. Fichrova R, Nakov L, Baleva $M$, et al: Sperm, nuclear, phospholipid and red blood cell antibodies and isotype RF in infertile couples and patients with autoimmune rheumatic diseases. Am J Reprod Immunol 36: 309-16, 1996.

20. Mathur S, Baker ER, Williamson HO, Derrick FC, Teague $\mathrm{KJ}$, Fudenbe HH: Clinical significance of sperm antibodies in infertility. Fertil Steril 36: 486-95, 1981.

21. Coppola A: Endocrine diseases and male infertility. Minerva Med 88: 355-63, 1997.

22. Cai L, Hales BF, Robaire B: Induction of apoptosis in the germ cells of adult male rats after exposure to cyclophosphamide. Biol Reprod 56: 1490-7, 1997.

23. Masala A, Faedda R, Alagna S, et al: Use of testosterone to prevent cyclophosphamide-induced azoospermia. Ann Intern Med 126: 292-5, 1997.

24. O'Morain C, Smethurst P, Dré Cj: Reversible male infertility due to sulphasalazine: studies in man and rat. Gut 25: 1078-84, 1984.

25. Lazowski Z, Janczewski Z, Polowiec Z: The effect of alkylating agents on the reproductive and hormonal testicular function in patients with rheumatoid arthritis. J Rheumatol 11: 49-54, 1982.

26. Aguilar-Mahecha A, Hales BF, Robaire B: Chronic cyclophosphamide treatment alters the expression of stress response genes in rat male germ cells. Biol Reprod 66: 1024-32, 2002.

27. Tabbara KF: Chlorambucil in Behcet's disease. A reappraisal. Ophtalmol 90: 906-8, 1983.

28. Chatzinoff M, Guarino JM, Corson SL, Batzer FR, Friedman LS: Sulfasalazine-induced abnormal sperm penetration assay reserved on changing to 5 -aminosalicylic acid enemas. Dig Dis Sci 33: 108-10, 1998.

29. Sharma RK, Kalla NR: Spermatozoal abnormalities and male infertility in the rat following sulfasalazine treatment. Int J Fertil Menopausal Stud 39: 347-54, 1994.

30. Steeno OP: Side-effects of salazopyrin on male fertility. Eur J Obstet Gynecol Reprod Biol 18: 361-4, 1984.

31. Fukushima T, Kato M, Adachi T, et al: Effects of sulfasalazine on sperm acrosome reaction and gene expression in the male reproductive organs of rats. Toxicol Sci 85: 675-82; 2004.

32. Baldi E, Luconi M, Bonaccorsi L, et al: Intracellular events and signaling pathways involved in spem acquisition of fertilizing capacity and acrosome reaction. Front Biosci 1;5: 110-23, 2000.

33. Sussman A, Leonard JM: Psoriasis, methotrexato and oligospermia. Arch Dermatol 116: 215-7, 1980

34. French AE, Koren G: Effect of methotrexate on male fertility. Can Fam Physician 49: 577-8, 2003.

35. Johnson FE, Farr SA, Mawad M, Woo YC: Testicular cytotoxicity of intravenous methotrexate in rats. J Surg Oncol 55: 175-8, 1994.

36. Haimov-Kochman R, Ben-Chetrit E: The effect of colchicine treatment on sperm production and function: a review. Hum Reprod 13:360-2, 1998.

37. Ehrenfeld M, Levy M, Margalioth EJ, et al: The effects of long-term colchicine therepy on male fertility in patients with familial Mediterranean fever. Andrologia 18: 420-6, 1986.

38. Ben-Chetrit E, Levy M: colchicine prophylaxis in familial Mediterranean fever: reappraisal after 15 years. Semin Arthritis Rheum 20: 241-6, 1991.
39. Levy M, Yaffe C: Testicular function in patients with familial Mediterranean fever on long-term colchicine treatment. Fertil Steril 29: 667-8, 1978.

40. Yu T: The efficacy of colchicine prophylaxis in articular gout- a reappraisal after 20 years. Semin Arthritis Rheum 12: 256-64, 1982.

41. Merlin HE: Azoospermia caused by colchicine. A case report. Fertil Steril 23: 180-1, 1972.

42. Fukutani K, Ishida H, Shinohara M, et al: Suppression of spermatogenesis in patients with Behcet's disease treated with cyclophosphamide and colchicine. Fertil Steril 36: 76-80, 1981.

43. Mzushima Y, Matsumura N, Mori M, et al: Colchicine in Behçet's disease. Lancet 2(8046): 1037, 1977.

44. Sarica K, Suzer O, Gurler A, et al: Urological evaluation of Behçet patients and the effect of colchicine on fertility. Eur Urol 27: 39-42, 1995.

45. Mendonça LL, Khamashta MA, Nelson-Piercy C, Hunt BJ, Hughes GR: Non-steroidal anti-inflammatory drugs as a possible cause for reversible infertility. Rheumatology (Oxford) 39: 880-2, 2000.

46. Kreysel CO: Value of prostaglandins in andrology. Fortschr Med 112: 203-6, 1994.

47. Kennedy JH, Korn N, Thurston RJ: Prostaglandin levels in seminal plasma and sperm extracts of the domestic turkey, and the effects of cyclooxygenase inhibitors on sperm mobility. Reprod Biol Endocrinol 1: 74, 2003.

48. Conte D, Nordio M, Romaneli F, et al: Role of seminal prostaglandins in male fertility. Effects of prostaglandin synthesis inhibition on spermatogenesis in man. J Endocrine Invest 8: 289-91, 1985.

49. Barkay J, Harpaz-Kerpel S, Ben-Ezra S, Gordon S, Zuckerman $\mathrm{H}$ : The prostaglandin inhibitor effect of antiinflammatory drugs in the therapy of male infertility. Fertil Steril 42: 406-11, 1984.

50. Moskovitz B, Lin R, Nssar S, Levin DR: Effect of diclofenac sodium on spermatogenesis of infertile oligospermic patients. Eur Urol 15: 395-7, 1988.

51. Sands BE: Why do anti-tumor necrosis factor antibodies work in Crohn's disease? Rev Gastroenterol Disord 4: S10-7, 2004.

52. Catrina AI, Trollmo C, af Klint E, et al: Evidence that anti-tumor necrosis factor therapy with etanercept and infliximab induces apoptosis in macrophages, but not lymphocytes, in rheumatoid arthritis joints: extended report. Arthritis Rheum 52: 61-72, 2005.

53. Bullimore DW: Endometriosis is sustained by tumor necrosis factor-alpha. Med Hypotheses 60: 84-8, 2003.

54. Eisermann J, Register KB, Strickler RC, Collins JL: The effect of tumor necrosis factor on human sperm motility in vitro. J Androl 10: 270-4, 1989.

55. Masuda H, Fujihira S, Ueno H, et al: Ultrastructural study on cytotoxic effects of cyclosporine A in spermiogenesis in rats. Med Electron Microse 36: 183-91, 2003.

56. Janssen NM, Genta MS: The effects of immunosuppressive and anti-inflammatory medications on fertility, pregnancy, and lactation. Arch Intern Med 160: 610-9, 2000.

57. Piediferro G, Colpi EM, Castiglioni F et al: Male contraception. Minerva Ginecol 56: 469-78, 2004.

58. Hoesl CE, Saad F, Poppel M, et al: Reversible, non-barrier male contraception: status and prospects. Eur Urol 48: 712-3, 2005. 